\section{Transapical Transcatheter Aortic Valve Implantation: The Berlin Experience}

\begin{abstract}
Transcatheter aortic valve implantation (TAVI) has been recently introduced as a means to treat patients with severe aortic valve stenosis at high risk or rejected for conventional surgery. In the present manuscript we summarize the Deutsches Herzzentrum Berlin (DHZB) experience with transapical TAVI starting from the initial steps for adequate TAVI team building and training and focusing on the technical issues and results in the first 500 operated patients. We will also comment upon the future of TAVI in the light of the results from large recent multi-center trials.
\end{abstract}

\section{KEY WORDS}

TAVI, TAVI team, surgical complications, future of TAVI.

DOI: $10.7251 / S M D 1202079 D$

(Scr Med 2012;43:79-84)

\section{Giuseppe D'Ancona, Miralem Pasic, Thorsten Drews, Semih Buz, Stephan Dreysse, Axel Unbehaun, Roland Hetzer}

Deutsches Herzzentrum Berlin, Germany

\section{Correspondence}

Professor Miralem PASIC

Deutsches Herzzentrum Berlin

Augustenburger Platz 1

D-13353 Berlin

Germany

Telephone number: +4930 45932108

Fax number: $\quad+493045932018$

E-mail: pasic@dhzb.de

Submitted: Sempember 01, 2012 Accepted: September 2O, 2012
Aortic valve stenosis (AVS) is the most common valvular cardiac pathology in Europe and North America. Its prevalence increases progressively with aging, and it presents as calcified aortic stenosis in 2-7\% of the population aged $>65$ years. ${ }^{1}$ Surgical indications for aortic valve replacement (AVR) are well defined in the international guidelines where it is specified that AVR should be performed only in symptomatic patients with severe AVS or in asymptomatic patients whenever there is an objective and subsequent left ventricular dysfunction. ${ }^{1}$

Although the results of surgical AVR are satisfactory in both the perioperative and long term follow-up phases, the perioperative risks increase for elderly patients and for those with complex comorbid profiles, which includes the vast majority of patients referred for AVR. Moreover, many patients with severe symptomatic AVS are not referred to surgical treatment because the examining physician considers surgery too traumatic and a prohibitive mortality risk. $^{2}$

Conventional AVR requires full sternotomy, heart lung machine adoption, cardiac arrest (cardioplegia), and "openheart" replacement of the native aortic valve. Despite excellent results achieved by conventional AVR in the past, it is mandatory to consider alternative interventions for a less invasive procedure. In this context, trans-catheter aortic valve implantation (TAVI) was recently introduced to treat patients with severe AVS that were rejected or considered too high risk for conventional surgery. Ten years after the first human implant, ${ }^{3}$ TAVI is a well accepted technique for treatment of over 35.000 patients worldwide.

Multicenter prospective randomized trials recently demonstrated the superiority of TAVI over conventional medical therapy for management of those patients with severe AVS who were rejected for standard surgery. ${ }^{4}$ In addition, TAVI is not inferior to conventional surgery for treatment of high risk surgical candidates (PARTNER trials). ${ }^{5}$ The benefits of this innovative technique remain consistent even at mid-term follow-up. ${ }^{6}$

We will summarize the Deutsches Herzzentrum Berlin (DHZB) experience with transapical TAVI starting from the establishment of DHZB TAVI team building and training principles in 2008 and moving towards the acquisition of a standardized patient selection and operative protocol. In the second part, we present results obtained with the first 500 patients treated via this revolutionary surgical operation. 


\section{Building and training the TAVI team}

Since the beginning of our experience with TAVI in 2008, the DHZB policy has been to structure two TAVI teams with complementary and synergic action: the core team and the virtual team. The core team consists of two anesthesiol-ogists with expertise in trans-esophageal echocardiography, five surgeons from four different surgical generations and two experienced cardiologists. The virtual team consists of about 50 persons involved in the chain of treatment of TAVI patients from the beginning to the end of hospitalization and in the out-patient follow-up.

A computer simulator, training by dry runs, and visiting centers that performed procedural life-case demonstrations provided procedural preparation. After these early theoretical stages, a first phase of proctoring was mandatory for the initial phases of the program building. External proctoring for the first cases was applied by pioneering experts, depending on the type of TAVI procedure. ${ }^{3,7}$ This proctoring was continued by occasional and repeated visits by other experts in order to exchange experience, improve details of the procedure and identify possible systemic errors inherent within the TAVI process.

After external proctoring stages, internal proctoring was managed as a self-appraisal relationship with regard to surgical experience between the team members, where opinions were exchanged in an open discussion during evaluation of the TAVI processes. This internal proctoring involved the most experienced surgeon/cardiologist ("senior proctors"); it was then gradually taken over by other members of the team ("younger proctors"), according to their surgical experience. This allowed those who were taught to "be proctor and proctored." During the initial phase, senior proctors performed all procedures if possible complications were expected due to patients' clinical condition (e.g. poor LVEF, cardiogenic shock, age $\geq 90$ years, special situations).

\section{Dhzb clinical and procedural policies for TAVI patients selection and procedural planning}

Inclusion Criteria. As previously emphasized, TAVI is proposed as a novel form of treatment for patients rejected from or at prohibitive risk for conventional aortic surgery to correct symptomatically severe AVS. Since the beginning of our experience at DHZB we have applied a "no exclusion" policy for referring patients to TAVI.

In fact, our idea is that every high-risk patient, including those with poor left ventricular ejection fraction (LVEF $=10-20 \%)$ or cardiogenic shock or those requiring a rescue procedure, should be considered for TAVI; exceptions would be those with endocarditis or too large annuli. No patient should be refused because of co-morbidities.
Patients are evaluated and accepted for TAVI according to contemporary accepted criteria that emphasize the importance of a predicted high mortality score (Euro-SCORE $>20 \%$, STS-score $>10 \%$ ). We believe that an institutional evaluation should be performed on the basis of the single center experience with conventional AVR and TAVI. Furthermore, some patients who do not fulfill the high risk classification criteria may be accepted for TAVI for technical surgical reasons (e.g. porcelain aorta) or because of a high risk for conventional operation due to factors not covered by the risk scores (e.g. liver cirrhosis, malignancy, or in special situations such as in patients with assist devices).

The reason(s) must be widely acceptable and must be clearly formulated and documented. We consider only active or recent endocarditis to be an absolute contraindication for TAVI. Obviously, an annulus size exceeding the recommendations of the valve manufacturers would also preclude TAVI.

Valve Sizing and Prosthesis Selection. We have developed a standardized system for evaluating native AV annulus size that allows us to select the appropriate trans-catheter prosthesis. The aortic annulus is assessed by preoperative trans-thoracic echocardiography, multislice computed tomography and intra-operative trans-esophageal echocardiography. A two mm over-sized valve is generally used when adopting the Edwards SAPIEN valve (Edwards SAPIEN THV, Edwards Lifesciences, Irvine, California). This device is the most frequently used trans-catheter aortic valve prosthesis at DHZB.

The Edwards SAPIEN heart-valve system consists of a trileaflet bovine pericardial valve and a balloon-expandable, stainless steel support frame (Fig. 1). Generally, we choose a valve size of $23 \mathrm{~mm}$ for aortic valve annuli smaller than $21 \mathrm{~mm}$, a $26-\mathrm{mm}$ prosthesis for annuli between 21 $\mathrm{mm}$ and smaller than $24 \mathrm{~mm}$, and a $29 \mathrm{~mm}$ valve for annuli between $24 \mathrm{~mm}$ and smaller than $27 \mathrm{~mm}$. A native aortic annulus of $19 \mathrm{~mm}$ diameter is the lower limit orientation value for the $23-\mathrm{mm}$ valve. In borderline cases, the decision is made on an individual basis, taking into account

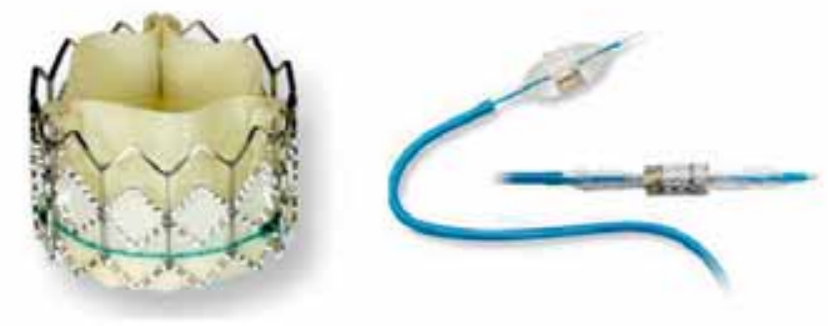

Figure 1. Edwards SAPIEN valve (Edwards SAPIEN THV, Edwards Lifesciences, Irvine, California) consists of a trileaflet bovine pericardial valve and a balloon-expandable, stainless steel support frame. 
additional factors. These include: the distances from the annulus to the coronary artery ostia, the shape of the annulus (oval versus circular), the amount of material in the leaflets, aortic diameters at the level of the sinuses of Valsalva, the sino-tubular junction, the ascending aorta, and the extent of calcification in structures, such as the left ventricular outflow tract, anterior mitral leaflet, and aortic valve leaflets themselves. ${ }^{8}$

Intraoperative phases for transapical TAVI. Since the beginning of our experience, TAVIs have been done in our special operating room (Fig. 2) that combines a catheter laboratory with all equipment and conditions necessary for surgery and sterile valve preparation before implantation; it includes requisite equipment for anesthesia, appropriate lighting, and a heart-lung machine. ${ }^{8}$ All operations

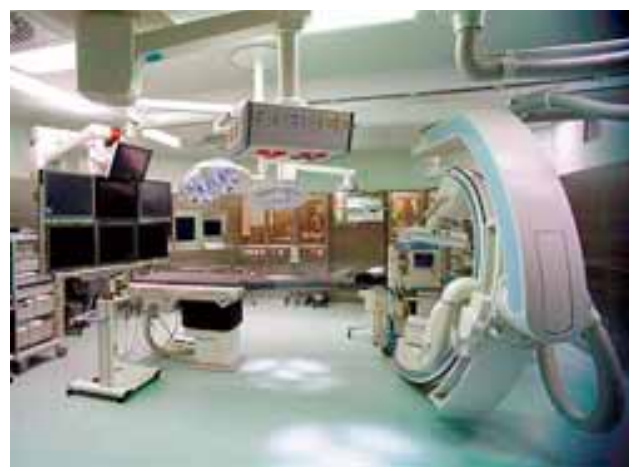

Figure 2. Hybrid operating room at the DHzB. This is a special operating room that combines a catheter laboratory with the preconditions necessary to perform surgery and sterile valve preparation before implantation, anesthesiologic equipment, appropriate lighting, and the heartlung machine. (From Pasic M, Unbehaun A, Dreysse $S$, et al. Transapical aortic valve implantation in 175 consecutive patients: excellent outcome in very high-risk patients. J Am Coll Cardiol 2010;56:813-20.)

are performed under general anesthesia with fluoroscopy and echocardiography guidance.

The patient is placed in a supine position, and a small anterior thoracotomy is performed 2 to $3 \mathrm{~cm}$ below the left mammary groove. The left ventricular apex is more easily reached, and the procedure more comfortably performed, when the chest is entered at the $6^{\text {th }}$ or $7^{\text {th }}$ intercostal space. In this way, the amount of chest retraction and dissection can be reduced. In any case, the correct position of the left ventricular apex is previously evaluated by chest computed tomography, and the actual position of the apex is checked digitally by the operator from a small opening in the intercostal space before extending the thoracotomy. ${ }^{8}$

Once the chest is entered, the pericardium is opened and the pericardial cradle is suspended to the thoracotomy

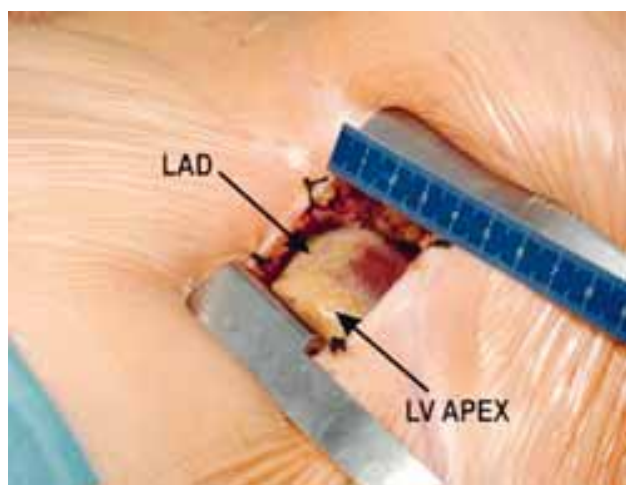

Figure 3. LV Apex exposure and preparation for TAVI through a small left anterior thoracocotmy in the $6^{\text {th }}$ $7^{\text {th }}$ intercostal space. LAD: Left anterior Descending coronary artery. (From Pasic M, Unbehaun A, Dreysse S, et al. Transapical aortic valve implantation in 175 consecutive patients: excellent outcome in very high-risk patients. J Am Coll Cardiol 2010;56:813-20.)

cutaneous edges. At this stage, the left ventricular apex is identified along with its anatomical boundaries (Fig. 3). Two polypropylene purse strings (either 3-0 or 2-o) are placed in an area of transaction between the left ventricular apex and the anterior wall of the left ventricle. The purse strings are reinforced with equine pericardium pledgets. At this stage a diagnostic pigtail is placed in the aortic root and pushed within the deepest aortic sinus to perform the first aortography. The ideal radiographic projection is to have the nadirs of two aortic sinuses (normally the left and right sinuses) lined up. When all three sinuses are visible in the same projection, their nadirs should line up. This condition is usually achieved with a radiographic projection of around 15 degrees left anterior oblique and 15 degrees cranial. Once the ideal projection is achieved, heparin is administered (100 IU/kg of body weight) and the left ventricular apex is punctured with a needle. A guide-wire is then passed through the aortic valve under fluoroscopy and echocardiography guidance. A small bore introducer ( $14 \mathrm{~F}$ ) is passed over the wire and through the valve. An exchange of the initial standard guide-wire is made with a stiff guide-wire protected by an angiography catheter. The valvuloplasty balloon is passed over the wire and through the calcified valve. An aortic balloon valvuloplasty is then done under rapid ventricular pacing (160 BPM).

After balloon deflation, the external pacing is discontinued and the small bore introducer removed. At this stage, a large bore (24-26 F) introducer is inserted into the apex over the stiff wire. The prosthetic valve, previously mounted on a balloon, is inserted in the large bore introducer and positioned within the native aortic valve annulus under fluoroscopy guidance. ${ }^{7,8}$ Aortography at this stage allows the initial selection of the valve position. Once the proper 
starting position has been identified, rapid ventricular pacing (16o BPM) is started. In this moment, while starting to slowly inflate the balloon, injection of 10 or $20 \mathrm{~mL}$ of contrast medium through the pigtail catheter (pulled back from the sinus of Valsalva 2 to $3 \mathrm{~cm}$ distally into the middle part of the ascending aorta just above the sinotubular junction) allows visualization (Fig 4). The balloon is initially expanded by about $30-40 \%$. The valve usually opens at the left ventricular end first. The position of the valve is corrected if necessary, and the balloon is further inflated to about $60-70 \%$. When the valve is $50-60 \%$ opened, fine correction of the valve position is still possible. The optimum positioning of the valve is obtained by precise alignment relative to the coronary artery ostia, native valve, and native aortic valve annulus. These structures are perfectly visible by this technique. ${ }^{9}$

Finally, the balloon is completely inflated, and the valve is deployed in the desired position (Fig. 4). At this stage, the balloon is deflated, external pacing is discontinued, and the balloon is retracted into the transapical sheath. After controlling the correct position and function of the newly implanted valve with the last aortography (Fig. 4), the introducer together with the balloon-catheter and the super-stiff guide-wire are pulled back and the apical purse strings are tied up.
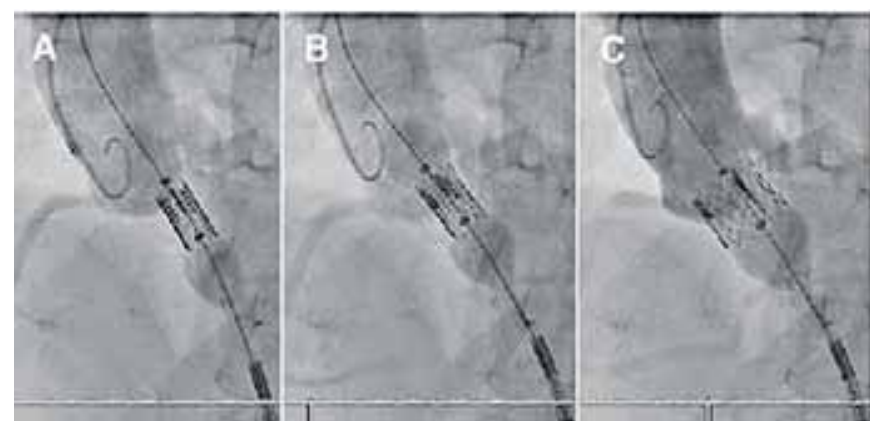

\section{Special conditions}

Use of femoro-femoral cardiopulmonary bypass (CPB). Our DHZB policy states that elective normothermic femoro-femoral CPB should be considered in patients with low LVEF (10-20\%), cardiogenic shock or rescue procedure, as well as in patients with significantly enlarged right ventricle (RV) with poor RVEF and severe pulmonary hypertension. ${ }^{10-12}$

Prophylactic use of intra-aortic balloon pump (IABP). IABP should be considered in patients with poor LVEF (10-20\%) and in patients in cardiogenic shock. ${ }^{10-12}$

Simultaneous treatment of combined coronary artery disease (CAD). Simultaneous elective percutaneous coronary intervention (PCI), or coronary artery bypass grafting if PCI is not possible, is considered for patients with concomitant CAD. Only the most relevant coronary artery stenosis is treated..$^{13-16}$

"Valve-in-valve" concept for degenerated biological valve prosthesis. This concept may be considered in high-risk patients as a therapeutic or a palliative approach. ${ }^{17,18}$

Figure 4. Valve deployment with angiographic monitoring. The starting position of the valve is intentionally chosen to be lower than usual because of a short distance from the aortic annulus to the ostium of the dominant right coronary artery $(8 \mathrm{~mm}$ as assessed by computed tomography). The balloon is only slightly inflated, the valve deployment begins, and angiography is performed (A). Then the valve position is corrected by pushing the valve slightly forwards into the aorta before full deployment (B). Concomitant angiography enables excellent visualization of the important anatomic factors in the proximal ascending aorta throughout the valve deployment and immediately after the valve is in its definitive position (C, D, E). In this particular case of high risk for occlusion of the right coronary artery, the valve is positioned very precisely and slowly and deployment requires a second injection of the contrast medium into the ascending aorta (D). Fluoroscopic view without contrast medium (F). (From Pasic M, Dreysse S, Drews T, et al. Improved technique of transapical aortic valve implantation: "the Berlin addition". Ann Thorac Surg 2010;89(6):2058-60.)

This slow and controlled release of the prosthesis is an original modification of the procedure by the DHZB TAVI team. It has been published in the medical literature as the "Berlin Addition." 9
Combined atrioventricular valve pathology. Concomitant significant functional atrioventricular valve pathology is not treated in combination with TAVI, but later on by conventional surgery if necessary. The exception is grade IV tricuspid valve regurgitation.

Post-implant paravalvular leakage or transvalvular regurgitation. Aortic regurgitation after valve 
implantation of grade 1 to 2 should be treated by additional balloon dilation of the valve and, if necessary, by implantation of a second valve. In the case of grade $\geq 2$, if it is not correctable, conventional surgical aortic valve replacement should be considered. ${ }^{19}$

Intra-procedural bleeding of unknown origin. Any arterial bleeding with no identifiable cause should be considered as a suspected annulus rupture. It requires immediate institution of CPB, and a median sternotomy should be performed, even in patients who are considered formally "inoperable" or "not suitable for conventional surgery." Standard aortic valve replacement is required, and, in the case of myocardial rupture, the left ventricular outflow tract (LVOT) should be reconstructed with an over-sized pericardial patch. No attempt should be made to close the ruptured left ventricle from the outside. ${ }^{20}$

\section{Dhzb results in the first $\mathbf{5 0 0}$ transapical TAVI}

This section presents results from the first 500 patients treated by trans-apical TAVI at the DHZB. All patients have been treated according to the indications and institutional protocols as discussed above.

The study cohort consists of 311 (62\%) female and 189 (38\%) male patients. The mean age was $79.5 \pm 8.1$ years (median 80.6 years, range 28.9-98.9 years). The mean logistic EuroSCORE (European System for Cardiac Operative Risk Evaluation) of the study cohort was $36.3 \pm 20.9 \%$ (median 30.4\%, range 4.1 to 96.7) and the mean STS PROM was $16.7 \% \pm 14.2 \%$ (median $12.2 \%$, range 1.2 to 89.5 ). There were 28 (5.6\%) patients with cardiogenic shock during the study period. The mean follow-up was $458.2 \pm 368.1$ days with a range from o (death during the procedural day) to 1363 days with a total of 628 patient years. At the time of the last data collection $374(74.8 \%)$ patients were alive and 126 (25.2\%) patients had died during the follow-up.

Surgical complications directly due to the procedure included surgical revision of apical pseudoaneurysm in two patients, revision for bleeding in eight patients, one iatrogenic aortic dissection (treated by transapical placement of an uncovered aortic endostent; patient survived), one valve migration. Annular rupture occurred in six patients throughout the study period.

The overall 30-day mortality for the entire study cohort of 500 patients was less than $5 \%$, with 23 deaths among 500 patients. The 30-day mortality for patients without preoperative cardiogenic shock was 4.0\% (19 deaths among 472 patients). The overall 6 month, 1 year and 2 year survival rates were $83.9 \pm 1.7 \%, 80.1 \pm 1.9 \%$ and $68.4 \pm 2.7$ for the whole group.

\section{Epilogue}

Since the first procedure in 2002, ${ }^{3}$ TAVI has become standardized, with over 35,000 implants performed all over the world. Our own results with transapical TAVI along with those from multicenter prospective randomized trials using transfemoral and transapical TAVI ${ }^{4-6}$ clearly indicate an encouraging midterm outcome along with acceptable perioperative mortality and rate of complications.

Results from the PARTNER trial reveal similarly high mortality at 2 years with TAVI and with surgical aortic valve replacement. In fact, follow-up mortality and complications rates presumably relate to the complex comorbid profile of elderly patients with symptomatic severe AVS. ${ }^{6}$

Perioperative results may impact follow-up outcome independently. In this context, residual aortic valve regurgitation after TAVI has been underestimated in the past. Paravalvular regurgitation is a design limitation of transcatheter aortic valves. This problem is most often secondary to incomplete and heterogeneous circumferential apposition of the prosthesis within the native annulus.

Recent scientific evidence ${ }^{6}$ supports our DHZB policy of not accepting residual aortic insufficiency greater than two. In fact, even mild paravalvular aortic regurgitation seems to be independently associated with increased late mortality. ${ }^{6}$ Avoiding valve under sizing and employing a patient's tailored post-TAVI re-dilation of the released transcatheter prosthesis can prevent this problem.

The future of TAVI. The successful application of TAVI in high risk patients ensures that it will, in the future, be used to treat even those individuals with low perioperative risk for conventional aortic valve surgery. The extension of TAVI indications will be aimed at minimizing the invasiveness of the procedure so as to further reduce the length and complexity of postoperative care. The SURTAVI Trial will start soon to evaluate the safety and efficacy of TAVI for treatment of symptomatic severe AS in subjects who are at intermediate risk for aortic valve surgery (STS mortality risk score $\geq 3 \%$ and $\leq 8 \%$ ).

In conclusion, TAVI is a revolutionary technique that has already changed the standard treatment of severe AVS. It should be emphasized that TAVI in general, and transapical TAVI in particular, is a "temptingly easy procedure (...) with 1001 sequences." ${ }^{8}$ Failure to respect one of the preoperative and/or perioperative steps may lead to abrupt catastrophe. Consistent results can be achieved and maintained in even the sickest patients, and the procedure can be eventually extended to a lower risk population through a systematic and structured TAVI training program. 


\section{Authorship statement}

G D'Ancona has written the manuscript and is a DHZB TAVI team member; $M$ Pasic has reviewed the manuscript and is the DHZB TAVI team and TAVI program Chief and senior surgeon; T Drews-S Buz- $S$ Dreysse- $A$ Unbehaun are DHZB TAVI team members; $R$ Hetzer is a DHZB TAVI team member and Chief of DHZB .

\section{Financial disclosure}

Prof. Pasic and Drs. Unbehaun, Drews, Buz, and Dreysse have been proctors to Edwards Lifesciences since July 2009. There are no other disclosures.

\section{References}

1. Bonow RO, Carabello BA, Chatterjee K, et al. 2008 Focused update incorporated into the ACC/AHA 2006 guidelines for the management of patients with valvular heart disease. Circulation 2008;118(15): e523-e661.

2. Lung B, Cachier A, Baron G, et al. Decision-making in elderly patients with severe aortic stenosis: why are so many denied surgery? Eur Heart J 2005;26:2714-20.

3. Cribier A, Eltchaninoff H, Bash A, et al. Percutaneous transcatheter implantation of an aortic valve prosthesis for calcific aortic stenosis: first human case description. Circulation. 2002, 10;106(24):3006-8.

4. Leon MB, Smith CR, Mack M, et al. Transcatheter aortic-valve implantation for aortic stenosis in patients who cannot undergo surgery. N Engl J Med 2010;363:1597-607.

5. Smith CR, Leon MB, Mack MJ, et al. Transcatheter versus surgical aortic-valve replacement in high-risk patients. N Engl J Med 2011;364:2187-98.

6. Kodali SK, Williams MR, Smith CR, et al. PARTNER Trial Investigators. Two-year outcomes after transcatheter or surgical aortic-valve replacement. N Engl J Med. 2012;366(18):1686-95.

7. Walther T, Dewey T, Borger MA, et al. Transapical aortic valve implantation: step by step. Ann Thorac Surg 2009;87:276-83.

8. Pasic M, Unbehaun A, Dreysse S, et al. Transapical aortic valve implantation in 175 consecutive patients: excellent outcome in very high-risk patients. J Am Coll Cardiol. 2010;56:813-20.

9. Pasic M, Dreysse S, Drews T, et al. Improved technique of transapical aortic valve implantation: "the Berlin addition". Ann Thorac Surg. 2010;89(6):2058-60.
10. Drews T, Pasic M, Buz S, et al. Elective femoro-femoral cardiopulmonary bypass during transcatheter aortic valve implantation: A useful tool. J Thorac Cardiovasc Surg. 2012;[Epub ahead of print] PMID: 22405673

11. Unbehaun A, Pasic M, Buz S, et al. Transapical aortic valve implantation in patients with severely depressed left ventricular function. J Thorac Cardiovasc Surg. 2012;143(6):1356-63.

12. D’Ancona G, Pasic M, Buz S, et al. Transapical transcatheter aortic valve replacement in patients with cardiogenic shock. Interact Cardiovasc Thorac Surg. 2012;14(4):426-30.

13. Pasic M, Dreysse S, Unbehaun A, et al. Combined elective percutaneous coronary intervention and transapical transcatheter aortic valve implantation. Interact Cardiovasc Thorac Surg. 2012;14(4):463-8.

14. Pasic M, Unbehaun A, Buz S, et al. Transapical aortic valve implantation and 'off-pump' arterial coronary bypass in a patient with a porcelain aorta. J Heart Valve Dis. 2011;20(6):711-4.

15. Mostafa AE, Geist V, Abdel-Wahab M. Ad-hoc percutaneous coronary intervention and transcatheter aortic valve implantation as a combined transfemoral procedure. J Invasive Cardiol. 2011;23(5):E102-5.

16. Conradi L, Seiffert M, Franzen O, et al. First experience with transcatheter aortic valve implantation and concomitant percutaneous coronary intervention. Clin Res Cardiol. 2011;100(4):311-6.

17. Drews T, Pasic M, Buz S, et al. Transapical aortic valve implantation after previous heart surgery. Eur J Cardiothorac Surg. 2011;39(5):625-30.

18. Drews T, Pasic M, Buz S, Unbehaun A. Trans-catheter aortic valve implantation after previous aortic homograft surgery. Eur J Cardiothorac Surg. 2011;40(6):1534-6.

19. Unbehaun A, Pasic M, Dreysse S, et al. Transapical aortic valve implantation: incidence and predictors of paravalvular leakage and transvalvular regurgitation in a series of 358 patients. J Am Coll Cardiol. 2012;59(3):211-21.

20. Pasic M, Unbehaun A, Dreysse S, et al. Rupture of the device landing zone during transcatheter aortic valve implantation: a life-threatening but treatable complication. Circ Cardiovasc Interv. 2012;5(3):424-32. 